\title{
A Hybrid Intelligent Method for Compensation of Current Transformers Saturation Based on PSO-SVR
}

\author{
Reza Taghipour Gorji', Seyyed Mehdi Hosseini ${ }^{*}$, Ali Akbar Abdoos¹, Ali Ebadi \\ ${ }^{1}$ Department of Electrical and Computer Engineering, Babol Noshirvani University of Technology, Shariati Av., \\ 47148-71167 Babol, Mazandaran, Iran \\ *Corresponding author, e-mail: mehdi.hosseini@nit.ac.ir
}

Received: 16 April 2020, Accepted: 01 July 2020, Published online: 25 January 2021

\begin{abstract}
The Current Transformers (CT) saturation may cause the protective relays mal-operation either non-recognition of internal fault or undesirable trip under external fault conditions. Therefore, compensation of CT saturation is very important for correct performance of protective schemes. Compensation of CT saturation by combination of signal processing methods and intelligent algorithms is a suitable solution to solve the problem. It decreases the probability of mal-operation and increases the reliability of the power system. In this paper, Support Vector Regression (SVR) method is employed to compensate the distorted secondary current due to CT saturation. In SVR method, despite the other methods such as MLPand ANFIS, instead of minimizing the model error, the operational risk error is considered as target function. In this method, by using Kernel tricks, a smart RBF neural network is obtained, so that all operational procedures will be optimized automatically. In this paper, an intelligent method based on Particle Swarm Optimization (PSO) algorithm is presented to determine the optimal values of SVR parameters. Due to the stability and robustness of this method in presence of noise and sudden changes in current, this method has a high accuracy. In addition, a sample power system is simulated using PSCAD software. Afterwards, current signals are extracted and fed to PSO-SVR algorithm, which is implemented in MATLAB environment. The obtained results show the preference of the proposed method in aspect of estimation accuracy as compared to some presented methods in the field of CT saturation detection and correction.
\end{abstract}

Keywords

Current Transformer (CT), saturation, Support Vector Regression (SVR), Particle Swarm Optimization (PSO), regression

\section{Introduction}

Current Transformers (CTs) are very important elements as they provide relays current signals proportional to primary current with a smaller range. Although CTs use iron core to maximize the flux linkage between primary and secondary windings, they are prone to saturation due to the non-linear magnetic characteristics of core.

The effective factors on the CT saturation are: short circuit level, short circuit occurrence location, fault resistance and fault angle. Among the above mentioned factors, the first three factors affect the severity of the fault current and the fourth factor determines the polarity and the magnitude of the exponential component of fault current. For operating conditions above the knee point of the magnetization curve, magnetizing current increases significantly. Therefore, the ratio error increases and results in distortion of the output signal. One way to limit this effect is either using CT with higher nominal characteristics or to use special algorithms to compensate this phenomenon. Since using CT with higher nominal characteristics is not cost-effective economically, soft compensation of CT saturation phenomenon in power system is an appropriate solution, which results in cost reduction and increase of the power system reliability [1].

A compensation method is suggested in [1] in which after estimating the magnetizing current, this current is added to the measured secondary current to correct the distorted secondary current. This algorithm works well for different fault conditions but the residual flux is considered zero before the fault occurrence. The proposed algorithm in [2] calculates the core flux and detects the saturation of CT under different conditions. However, in this method the initial flux is considered zero in the beginning of calculation, which is not appropriate assumption under real conditions. 
The different order of secondary current signal deviation is also applied to detect the saturation [3-8]. A combination of second-order derivation and zero-passing technique is also applied to improve the detection of saturation time internal. In this method, only saturation cycles are detected and the start and the end points of saturation time intervals are not determined [5]. In [8], the secondary current and its derivatives are used in order to compensate the saturation of current transformer. In [7] and [9], a method is presented to detect the saturation of CT based on third-order derivative of secondary current. In these papers, the effect of the anti-aliasing low-pass filter is also considered. A method for detection of saturation is suggested in [10] by calculating the mean and the variance of the fault. In [11], a method is presented for detection and compensation of the saturated secondary current by using the derivatives of secondary current and Newton backward differential formula. The proposed method is dependent on an index as the saturation detection criterion. This index is consistently compared with an expected value while the start point and the end point of saturation area can be detected. A low-pass filter is used to remove the noise and harmonics contents of the secondary current. This method is simulated by taking into account the residual flux of core, burden of CT as well as the DC component and the noise of the current signal. Regarding the advantages of differential methods such as simplicity of calculations and the possibility of their implementation in on-time applications, these methods have suffered from some difficulties such as very high dependency on sampling frequency, high sensitivity to noise, high dependency of detected output signal to the strength and weakness of the CT's core saturation [11].

Wavelet transform as a signal analysis tool within the domain of time and frequency can determine the beginning and the end points of saturation [12-14]. In wavelet transform based methods, the current signals are decomposed into two levels of approximation and detail in different stages by using some determined filters called mother wavelet [12]. Detail level including the contents of high frequency components, can be employed to recognize the saturation time interval. The main problem of wavelet transform and derivative based methods is noise sensitivity. Therefore, before applying these methods, the signal should be passed through the low-pass filters [13].

In many articles, Artificial Neural Networks (ANN) are utilized for detection and correction of CT saturation [15-17]. The current samples of CT secondary side are applied for training the neural network. In [18, 19], a method is suggested to detect the saturation of CT using ANN trained by Genetic Algorithm (GA). The main problem of ANN based method is that a large number of training data is needed during learning process. This method requires so many parameters, which should be set in order to achieve optimal and efficient structure [18].

In this paper, Support Vector Regression (SVR) method is presented for detection and correction of the CT secondary current in saturation conditions. SVRs have the capability to learn training patterns and can be utilized as either classifier or regression tool. Compared to ANN, SVRs have less adjustable parameters and simpler structure [20]. In this paper, Particle Swarm Optimization (PSO) method is presented to optimize the SVR model parameters values. This new structure is presented as PSO-SVR method.

\section{Test study}

As shown in Fig. 1, a part of a high voltage substation in Iran [21, 22], including a power transformer, grounding transformer and CTs is modeled in PSCAD software. The specification of the power transformer and CTs are presented by Tables 1 and 2, respectively. In order to model the residual flux, current sources are inserted in the primary side. To examine a wide range of faults, various faults were applied inside and outside the protected areas considering with different resistances.

It must be noted that the CTs are simulated based on the Jiles-Atherton (JA) model [21]. This model is based on the phenomenological model of a ferromagnetic core and the relationship between the magnetic moment and

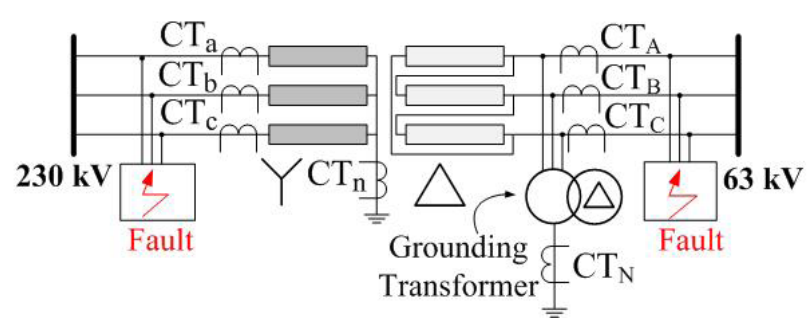

Fig. 1 Power system modeled in PSCAD

Table 1 Power Transformer Characteristics

\begin{tabular}{lc}
\hline Technical Data & Value \\
\hline Rated Power (MVA) & 160 \\
Rated Voltage Ratio (kV/kV) & $230 / 63$ \\
Connection & $\mathrm{Y} / \Delta$ \\
Frequency (Hz) & 50 \\
Leakage Reactance (p.u.) & 0.14 \\
No-Load Losses (p.u.) & 0.002 \\
Copper Losses (p.u.) & 0.004 \\
\hline
\end{tabular}


Table 2 CT specifications with 1 A secondary nominal current

\begin{tabular}{lcc}
\hline CT & HV Side & LV side \\
\hline Turn Ratio & $600 / 1$ & $2000 / 1$ \\
Cross Section $\left(\mathrm{m}^{2}\right)$ & $32.9 \times 10^{-4}$ & $10.36 \times 10^{-4}$ \\
Core Length $(\mathrm{m})$ & 0.848 & 0.548 \\
Sec. Winding Res. $(\Omega)$ & 4.3 & 7.77 \\
\hline
\end{tabular}

the intensity of the magnetic field. Currently used models, use mathematical techniques, which approximate the behavior of the hysteresis loop for recoil loops and renascence effects [22, 23]. For many studies, these models are quite adequate except where accurate values of renascence are required following a circuit interruption with a subsequent reclose into a live circuit. This feature is particularly important in relay studies where it is necessary to simulate successive faults due to reclosing of a permanent fault. During validation of the new model against recordings from a synthetic test plant, the shape of the loop in the shoulder area was also found to be critical in situations where the burden was small (as in digital relays) and the core carried residual flux. In situations where the burden is large, which leads to severe saturation, the exact shape of the loop was not critical. The latter situation is the one that most often used in simulation studies [23]. Jiles and Atherton used a soft magnetic material for their investigation and modeled the saturation characteristic using a modified Langevin function. In JA model, by solving the basic equations [21], model parameters can be determined by maximizing the accurate estimation of the curve of the magnetic CT core. Since the magnetizing curve is available, the above mentioned parameters can be determined by using random search methods. In this article, the PSO method is used to determine the JA parameters. CT core magnetizing curve is shown in Fig. 2 and JA model parameters can be found in Table 3 .

\section{Support Vector Regression (SVR)}

SVR method (Fig. 3) is one of the supervised learning methods, which is widely used to solve complicated data classification and regression problems [24]. The SVR algorithm can be easily implemented in other machine learning problems such as function fitting. Unlike other methods such as Multilayer Perceptron (MLP) and Adaptive Neuro-Fuzzy Inference System (ANFIS) that try to minimize the modeling error, in SVR method, operational risk is considered as target function. In this method, using Kernel core functions technique and by determining its parameters, indeed an intelligent Radial Basis Function

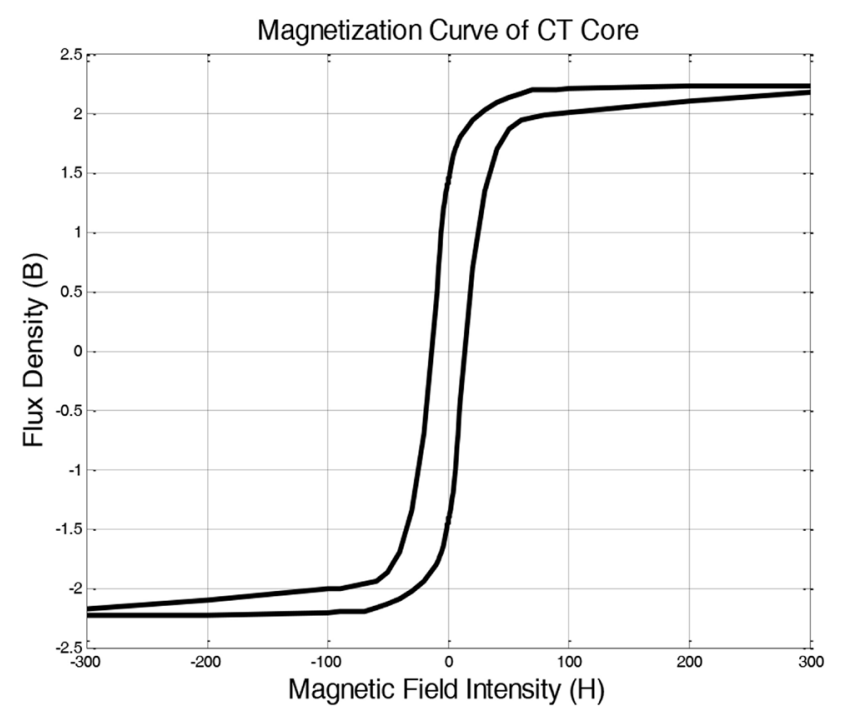

Fig. 2 Current Transformer magnetizing curve

Table 3 Parameters related to specific CT core by the JA

\begin{tabular}{lc}
\hline Item & Value \\
\hline Domain Flexing Parameter $(c)$ & 0.1537 \\
Domain Pinning Parameter $(k)$ & $2.55 \times 10^{-7}$ \\
Parameter to Adjust K with M $(\beta)$ & 0.97 \\
Inter-Domain Coupling $(\alpha)$ & $3.93 \times 10^{-6}$ \\
Coefficient 1 of Anhysteretic Curve $\left(\alpha_{1}\right)$ & 2896 \\
Coefficient 2 of Anhysteretic Curve $\left(\alpha_{2}\right)$ & 3054 \\
Coefficient 3 of Anhysteretic Curve $\left(\alpha_{3}\right)$ & 17245 \\
Coefficient 4 of Anhysteretic Curve $(b)$ & 2 \\
Saturation Anhysteretic Magnetization $\left(M_{s}\right)$ & $1.889 \times 10^{6}$ \\
\hline
\end{tabular}

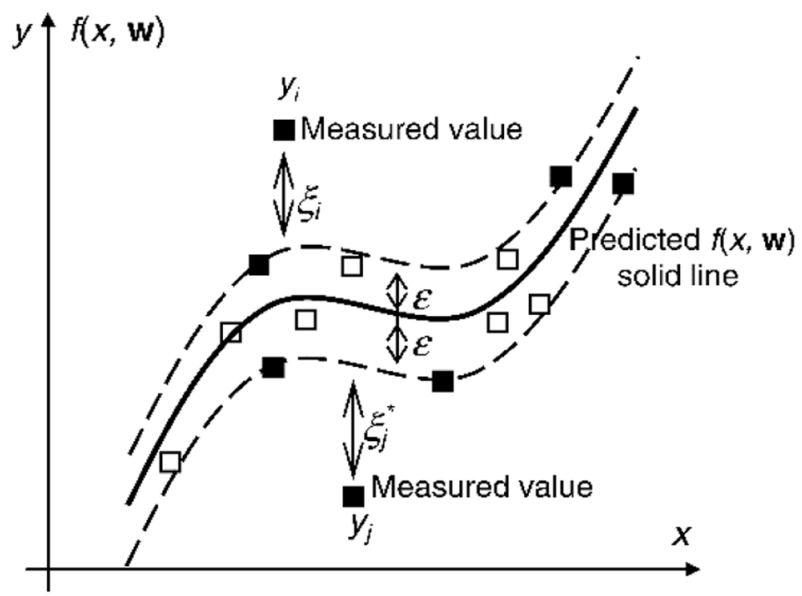

Fig. 3 The parameters used in the SVR method

(RBF) will be achieved, which all of the operational procedure will be automatically optimized. In the regression by SVR, the non-linear and complicated relationship between input and output is estimated [20]. In spite 
of pattern recognition problems that have discrete output (0 and 1), regression problems outputs are real numbers.

The basis of SVR performance in non-linear regression is to predict the target curve with the maximum confidence margin. To solve the equation and find the target regression curve, Quadratic Programming (QP) methods are used, which are well-known for solving the optimization problems. For solving non-linear problems with high dimensions, Lagrangean dual theorem is used to convert the desired minimization problem into its dual form [25].

The first step in creation of SVR model is to select a non-linear mapping algorithm as a kernel function through which the input vectors are mapped into a high dimensional space. In this space, simpler linear regressions can be replaced with complex non-linear regressions of the original input space [25]. To perform regression, it is assumed that $l$ pair of training data for learning process is as follows:

$$
\begin{aligned}
& D=\left\{[x(i)],[y(i)] \in R^{n} \times R, i=1,2, \ldots, l\right\} \\
& y=f(x, w)=w \times \varphi(x)+b .
\end{aligned}
$$

Equation (1) includes 1 pair of sample data $\left(x_{1}, y_{1}\right), \ldots,\left(x_{n}, y_{n}\right)$, in which the inputs of $n$ dimensional vectors of $x \in R^{n}$ and the outputs of unidimensional vector $y \in R$, are in the form of real numbers. In Eq. (2), $w$ is the matrix of the regression weight vector, $b$ is bias, $\phi(x)$ is a non-linear function by which $x$ is mapped into a high dimensional space. In the mapping process, a kernel function is constructed by $K\left(x, x^{\prime}\right)=\left(\phi(x) \times \phi\left(x^{\prime}\right)\right)$. Kernel functions which can be implemented in SVR method are very variant [26]. In this paper, Gaussian (RBF) kernel function is used to obtain optimal solution (i.e. Eq. (3)). The RBF kernel is generally applied most frequently because it has few adjustment parameters as well as a desired performance in high-volume data.

$$
K\left(x, x_{i}\right)=\exp \left(-\frac{\left\|x-x_{i}\right\|^{2}}{2 \gamma^{2}}\right),
$$

where $x_{i}$ is the input vector and $\gamma$ is the parameter of the RBF kernel function.

The obtained answer for the regression hyperplane of $f(x, w)=w \times \phi(x)+b$ will produce a non-linear regression hyperplane in the main input space. Total risk value and the norm of $w$ should simultaneously be minimized.

Therefore, a linear regression hyperplane and risk minimization is obtained employing Lagrangian techniques as follows: minimize

$$
R_{w, b, \xi, \xi^{*}}=\left[\frac{1}{2} w^{T} w+C \sum_{i=1}^{l}\left(\xi_{i}+\xi_{i}^{*}\right)\right]
$$

subject to

$$
\begin{aligned}
& y_{i}-\left(w^{T} \phi_{i}(x)+b\right) \leq \varepsilon+\xi_{i} \\
& \left(w^{T} \phi_{i}(x)+b\right)-y_{i} \leq \varepsilon+\xi_{i}^{*} \\
& \xi_{i}, \xi_{i}^{*} \geq 0 \\
& i=1,2, \ldots, N .
\end{aligned}
$$

In the above equations, $C$ is penalty factor, which determines the penalty value for the violation of samples from the allowed error of regression. $N$ is the number of the samples. $\varepsilon$ is the error tolerance parameter, which is considered 0.001 in this paper. $\xi_{n}$ and $\xi_{n}^{*}$ are positive auxiliary variables, which determine the violation level of training data from up and down limits of $\varepsilon . \xi_{n}$ represents the upper training errors subject to $\varepsilon$ and $\xi_{n}^{*}$ represents the lower training errors subject to $\varepsilon$.

$C$ and $\gamma$ are two main parameters in SVR method by RBF kernel implementation, which should appropriately be adjusted for desired performance of algorithm [27, 28]. Parameter $C$ expresses the cost value of violation penalty. The value $C$ is effective on the regression precision and the performance of SVR system. If $C$ is selected very large, the accuracy of regression will be high in the training phase, but in testing phase the accuracy will be very low. If $C$ is selected very small, the accuracy of training phase will be very low and the appropriate model will not be obtained. The parameter $\gamma$ has an effect more than that of $C$ in SVR model. High value of $\gamma$ causes over-fitting and very small value of it causes under-fitting. In this paper, PSO method is employed to optimize the SVR parameters value [29].

\section{The presented CT saturation detection model}

In this section, PSO-SVR hybrid model is presented for regression in high-dimensional data. In this method, the purpose is to enhance the precision of prediction and regression using determination of the appropriate structure of the input data and adjustment of RBF kernel and SVR parameters. This study employs the PSO technique for proper setting of adjustable parameters. Two other decision variables i.e. $C$ and $\gamma$ should also be determined. Firstly, the population of particles is initialized. Each particle has a random position within the $D$-dimensional space and a random velocity for each dimension. At second step, for each particle, the fitness i.e. the regression accuracy is 
calculated. If the fitness is better than the particle's best fitness, then the position vector is saved for the particle. If the particle>s fitness is better than the global best fitness, then the position vector is saved for the global best. Finally, the particle's velocity and position are updated until the termination condition is satisfied [30-33]. Fig. 4 shows the architecture of the developed PSO-based parameter determination approach for SVR model.

For preparation of input data, we collect the necessary information from primary and secondary current in the event of faults and the secondary current that has been distorted by saturation. Sampling frequency in this project is $2.5 \mathrm{kHz}$. To cover a wide range of faults, different faults at different times within the protection area and outside of it are applied by different resistances. In order to cover all of the fault conditions, 20 SVR systems are considered for CT saturation compensation.

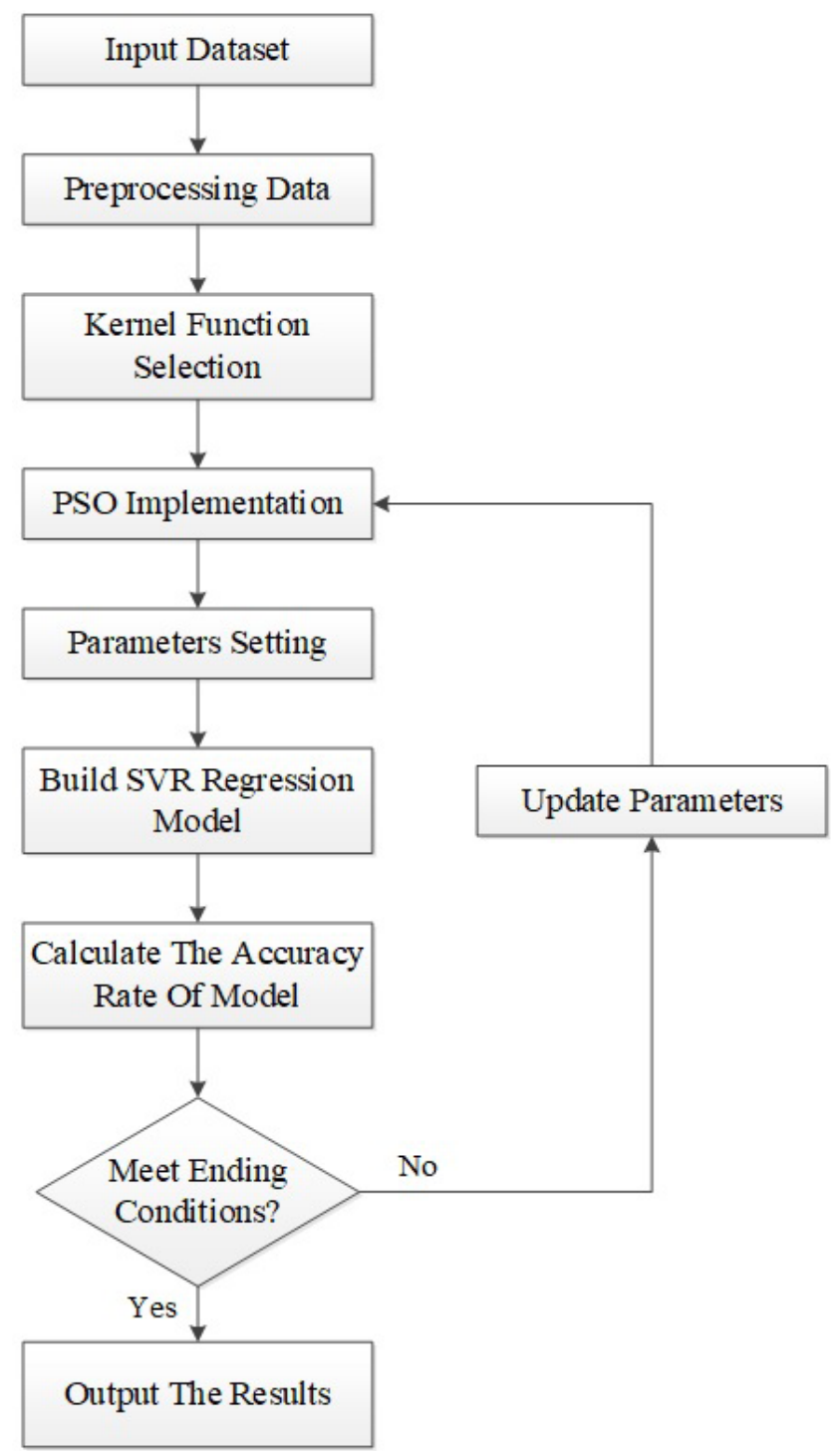

Fig. 4 The architecture of the proposed PSO-SVR method
Each SVR system designed for a short range of time constant value and each one includes different fault amplitude. For each SVR system, the input matrix $P$ and the desirable output $T$ is created for training stage. In the first moments after the fault occurrence, data are healthy in the secondary current signal before the saturation. By taking the first 8 healthy samples in secondary current, the first 7 samples is considered as the first column elements of $P$ matrix and the $8^{\text {th }}$ sample is considered as the first element of output matrix. Then, the second to eighth samples create the second column of the matrix $P$ and ninth sample create the second element of matrix $T$ and so on.

$\begin{aligned} P & =\left[\begin{array}{ccc}P_{11} & \ldots & P_{1 N} \\ \vdots & \ddots & \vdots \\ P_{s 1} & \ldots & P_{s N}\end{array}\right] \\ T & =\left[\begin{array}{lll}T_{11} & \ldots & T_{1 N}\end{array}\right]\end{aligned}$

In the next step, kernel function and its parameters as well as SVR parameters should be determined. The main advantage of this method is that there is a few adjustable parameters during training of the SVR system for regression. By choosing the RBF kernel function, $\gamma$ and $C$ parameters are determined by PSO method.

After determining the model coefficients and running the program, the Lagrange coefficients $\alpha$ and $\alpha^{*}$ will be calculated. Support vectors are those that $\left(\alpha-\alpha^{*}\right)$ is nonzero for them. In the next step bias coefficient $b$ will be calculated and finally, we have all the parameters of the model in Eq. (7).

$$
f(X, W)=W_{0}^{T} \times \Phi(X)+b=\sum_{i=1}^{l}\left(\alpha_{i}-\alpha_{i}^{*}\right) \times K\left(X_{i}, X\right)+b
$$

SVR system intelligently determines the number of RBF kernel functions, so results in the simple RBF model that causes the minimum time in running the model (Fig. 5). In this method, by using Kernel Trick we can achieve a smart RBF neural network that all operational procedures will be optimized automatically. In the classic model of RBF neural network, we have the neurons placement problem and determining the number of hidden neurons. However, in SVR method this problem is resolved by using support vectors theory.

In the final step, we test the SVR system with the testdata and calculate the prediction error indicators to evaluate the accuracy of model. In order to analyze the performance of the model, five indicators are determined including the Absolute Error (AE), Relative Error (RE), 


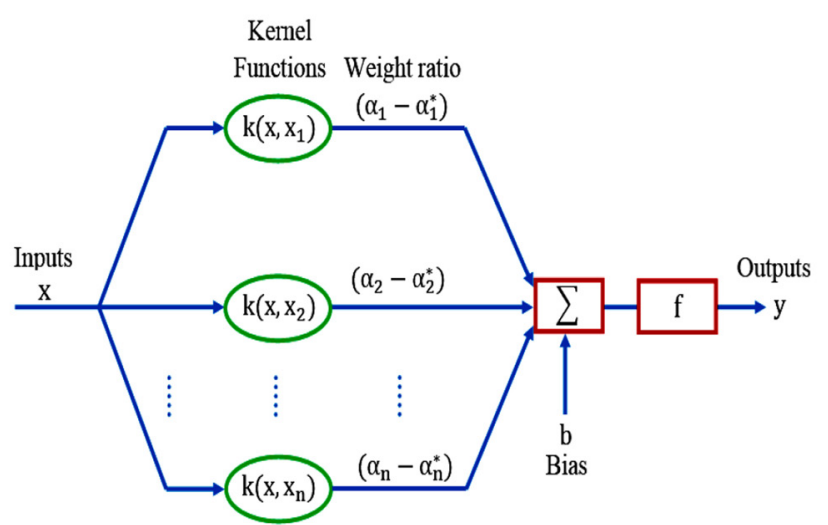

Fig. 5 SVR system structure and its similarity to the RBF neural network.

Mean Square Error (MSE), Root Mean Square Error (RMSE) and correlation coefficient $(R)$. These indicators were selected to evaluate the fitness and precision of the model. AE represents the deviation between monitoring and predicted values. RE is the ratio of $\mathrm{AE}$ and monitoring values reflecting the objective accuracy of measurement results. MSE and RMSE reflect the performance of the prediction model. Generally, the smaller the RMSE, the better the performance. $R$ represents the degree of linear relevance among the variables. The closer $R$ to 1 , the higher is the relevance. The expressions of these indicators is as follows where $y_{i}$ is the real value of the data set, $\hat{y}_{i}$ is predicted value, $\bar{y}$ is the average of the original data, and $n$ is the amount of data for the testing set.

$\mathrm{AE}=\left|y_{i}-\hat{y}_{i}\right|$

$\mathrm{RE}=\frac{\mathrm{AE}}{y_{i}} \times 100 \%$

$R=\sqrt{1-\frac{\sum_{i=1}^{n}\left(y_{i}-\hat{y}_{i}\right)^{2}}{\sum_{i=1}^{n}\left(y_{i}-\bar{y}\right)^{2}}}$

$\operatorname{MSE}=\frac{1}{n} \sum_{i=1}^{n}\left(y_{i}-\hat{y}_{i}\right)^{2}, \operatorname{RMSE}=\sqrt{\mathrm{MSE}}$

\section{Results and discussion}

Saturated current prediction using Artificial Neural Networks (ANN) and prediction error indicators for the fault current with $5.711 \mathrm{kA}$ amplitude and the time constant $89.17 \mathrm{~ms}$ is shown in Fig. 6. As you can see, the error of prediction is gradually increased and the anticipated signal cannot predict purpose signal correctly.

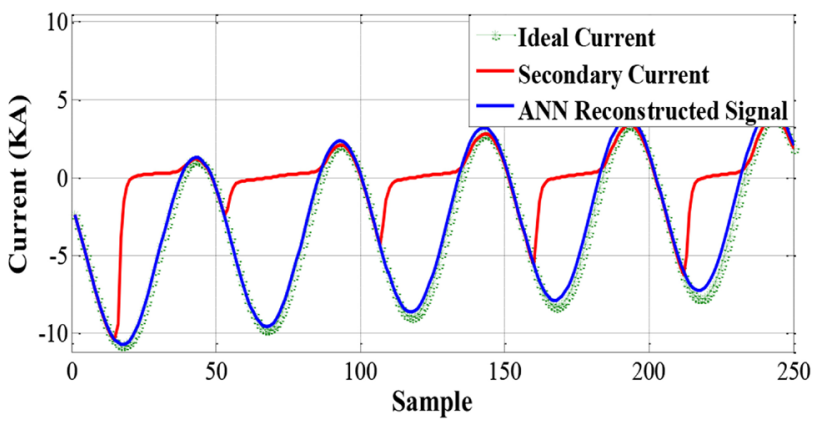

(a)

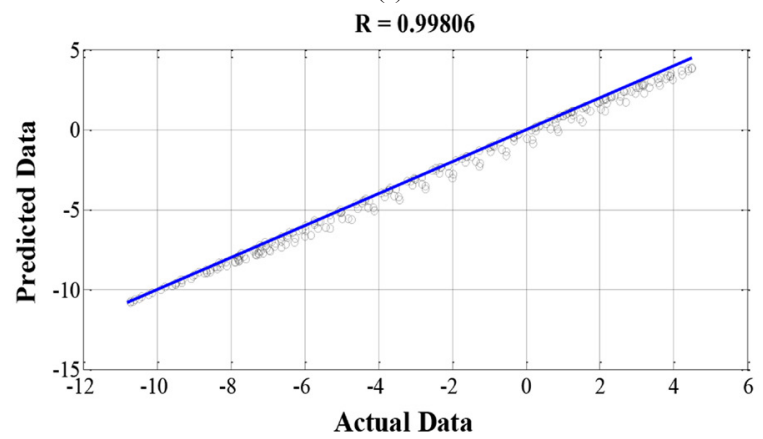

(b)

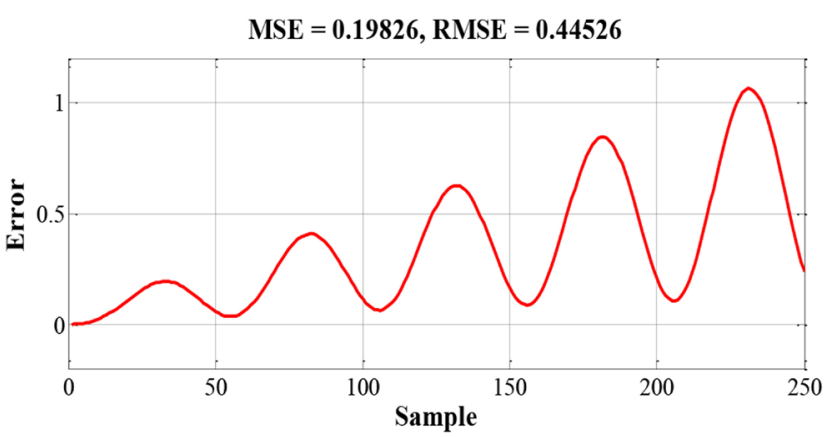

(c)

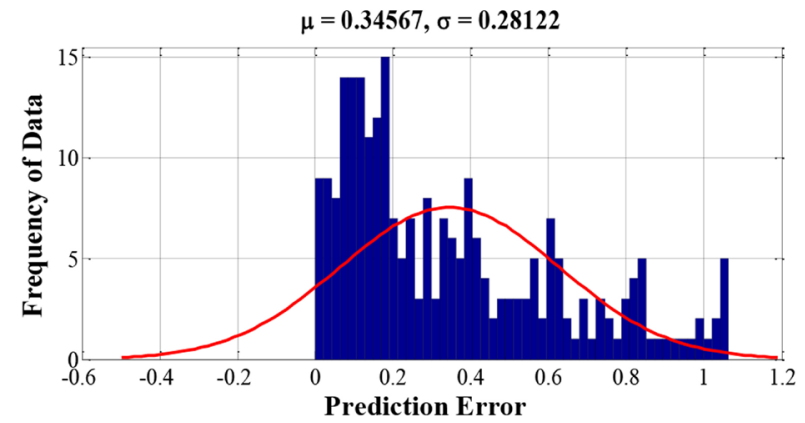

(d)

Fig. 6 ANN prediction for the fault current with $5.711 \mathrm{kA}$ amplitude and the time constant $89.17 \mathrm{~ms}$. (a) The primary current transferred to the secondary and predicted signal. (b) Correlation between actual and predicted data. (c) Changes of error and MSE and RMSE quantities. (d) Histogram of prediction error and its mean $(\mu)$ and variance $(\sigma)$.

The results of anticipated saturated current with ANN method provided in Table 4 for different values of waveform amplitude and time constant. In ANN method, 
Table 4 Prediction results by ANN method

\begin{tabular}{llccccc}
\hline $\begin{array}{l}\text { Fault } \\
\text { Current }\end{array}$ & & \multicolumn{5}{c}{ Prediction by ANN Method } \\
Prediction Error Indexes $\left(\times 10^{-3}\right)$ \\
$I_{m}(\mathrm{KA})$ & $\tau(\mathrm{ms})$ & $R(\%)$ & MSE & RMSE & $\mu$ & $\sigma$ \\
\hline 5.711 & 89.17 & 99.8 & 198.2 & 445.2 & 345 & 280 \\
5.671 & 25.7 & 99.9 & 1.9 & 143.7 & 39 & 20 \\
5.553 & 15.07 & 99.9 & 28.7 & 169.5 & 135 & 10 \\
\hline
\end{tabular}

we can reach low RMSE index level by designing ANN network properly. But selecting the structure of ANN and its parameters adjustment and the huge number of data that are needed for training ANN, are serious challenges. However, this method is highly sensitive to changes in the structure of the power system so that any changes can lead to a lack of efficiency and accuracy of this method in predicting the target signal.

Saturated current prediction using SVR that its parameters are determined by PSO algorithm, is shown in Fig. 7. For the fault current with $5.711 \mathrm{kA}$ amplitude and the time constant $89.17 \mathrm{~ms}$, the RMSE reached to 0.0182 that is very small and acceptable for practical applications. According to the tolerance of error in Fig. 7 (c), predicted signal has very small deviation from the target signal. By PSO-SVR hybrid method and the performance of the support vectors, the deviation of the predicted signal is limited to small quantities. The results of anticipated saturated current with PSO-SVR method provided in Table 5 for different values of waveform amplitude and time constant (Fig. 8). As shown, the error indices have small and desirable values.

\section{Conclusion}

In this paper, a new hybrid algorithm is presented based on SVR for compensation of CT saturation. There are a few adjustable parameters in the structure of SVR model such as Non-sensitive area parameter $(\varepsilon)$, penalty factor $(C)$ and parameters related to the kernel function. In this paper, PSO-SVR hybrid model is presented to determine the optimal values of adjustable parameters. The main advantages of the proposed intelligent method are low computational burden and high stability and robustness in the presence of noise and sudden changes in current samples. Because of few numbers of adjustable parameters and high training capabilities, the SVR method is an appropriate tool to compensate the fault current of CTs in saturation mode in real time implementation. The obtained results justify the applicability of the proposed method to effectively compensate the CT saturated secondary current for different operating conditions.

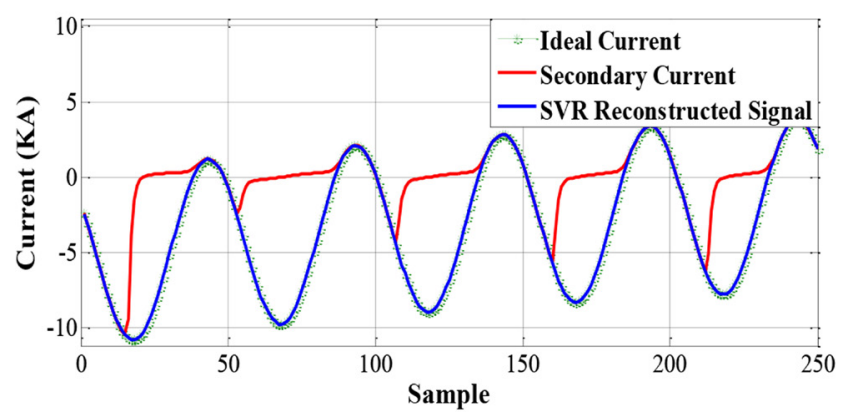

(a)

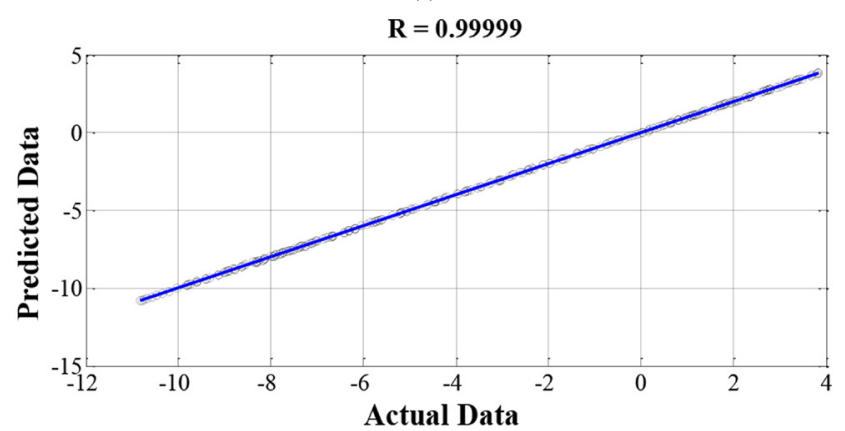

(b)

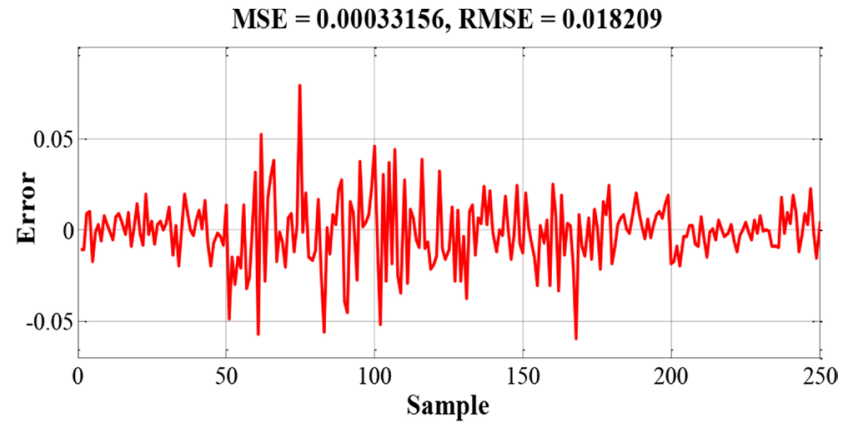

(c)

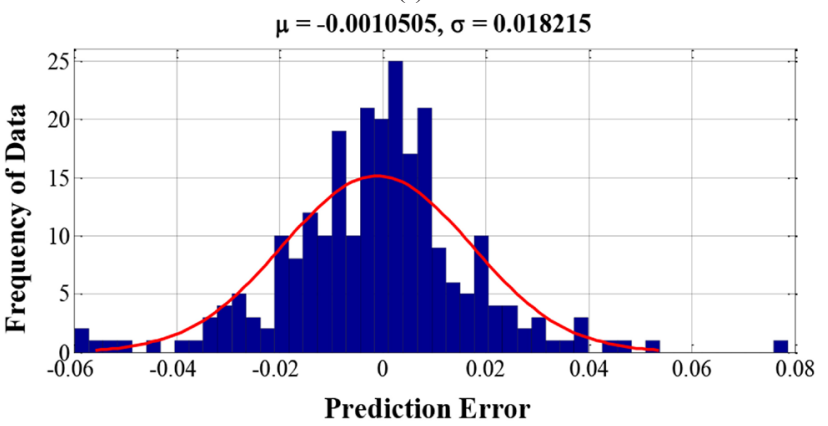

(d)

Fig. 7 PSO-SVR prediction for the fault current with $5.711 \mathrm{kA}$ amplitude and the time constant $89.17 \mathrm{~ms}$. (a) The primary current transfered to the secondary and predicted signal. (b) Correlation between actual and predicted data. (c) Changes of error and MSE and RMSE quantities. (d) Histogram of prediction error and its mean $(\mu)$ and variance $(\sigma)$.

\section{Acknowledgement}

The authors acknowledge the funding support of "Babol Noshirvani University of Technology" Grant program Numbers BNUT/390066/99 and BNUT/370199/99. 
Table 5 Prediction results by PSO-SVR method

\begin{tabular}{llccccc}
\hline $\begin{array}{l}\text { Fault } \\
\text { Current }\end{array}$ & \multicolumn{5}{c}{ Prediction by PSO-SVR Method } \\
$I_{m}(\mathrm{KA})$ & $\tau(\mathrm{ms})$ & $R(\%)$ & MSE & RMSE & $\mu$ & $\sigma$ \\
5.711 & 89.17 & 99.9 & 0.33 & 18.2 & -0.1 & 18 \\
5.671 & 25.7 & 99.9 & 0.23 & 15.3 & -0.5 & 15 \\
5.553 & 15.07 & 99.9 & 0.22 & 14.8 & -0.7 & 19 \\
\hline
\end{tabular}

\section{References}

[1] Kang, Y. C., Kang, S. H., Park, J. K., Johns, A. T., Aggarwal, R. K. "Development and hardware implementation of a compensating algorithm for the secondary current of current transformers", IEE Proceedings - Electric Power Applications, 143(1), pp. 41-49, 1996. https://doi.org/10.1049/ip-epa:19960040

[2] Kang,Y. C., Park, J. K., Kang, S. H., Johns, A. T., Aggarwal, R. K. "An algorithm for compensating secondary currents of current transformers", IEEE Transactions on Power Delivery, 12(1), pp. 116-124, 1997. https://doi.org/10.1109/61.568231

[3] Kang, Y. C., Lim, U. J., Kang, S. H., Crossley, P. "Compensation of the distortion in the secondary current caused by saturation and remanence in a CT", IEEE Transactions on Power Delivery, 19(4), pp. 1642-1649, 2004.

https://doi.org/10.1109/TPWRD.2004.835266

[4] Wiszniewski, A., Rebizant, W., Schiel, L. "Correction of Current Transformer Transient Performance", IEEE Transactions on Power Delivery, 23(2), pp. 624-632, 2008.

https://doi.org/10.1109/TPWRD.2008.915832

[5] Dashti, H., Sanaye-Pasand, M., Davarpanah, M. "Fast and Reliable CT Saturation Detection Using a Combined Method", IEEE Transactions on Power Delivery, 24(3), pp. 1037-1044, 2009. https://doi.org/10.1109/TPWRD.2009.2022666

[6] Hooshyar, A., Sanaye-Pasand, M., Davarpanah, M. "Development of a new derivative-based algorithm to detect current transformer saturation", IET Generation, Transmission \& Distribution, 6(3), pp. 207-217, 2012.

https://doi.org/10.1049/iet-gtd.2011.0476

[7] Kang, Y.-C., Ok, S.-H., Kang, S.-H. "A CT saturation detection algorithm", IEEE Transactions on Power Delivery, 19(1), pp. 78-85, 2004. https://doi.org/10.1109/TPWRD.2003.820200

[8] dos Santos, E. M., Cardoso, G., Farias, P. E., de Morais, A. P. "CT Saturation Detection Based on the Distance Between Consecutive Points in the Plans Formed by the Secondary Current Samples and Their Difference-Functions", IEEE Transactions on Power Delivery, 28(1), pp. 29-37, 2013.

https://doi.org/10.1109/TPWRD.2012.2220382

[9] Kang, Y. C., Ok, S. H., Kang, S. H., Crossely, P. A. "Design and evaluation of an algorithm for detecting current transformer saturation", IEE Proceedings - Generation, Transmission and Distribution, 151(1), pp. 27-35, 2004.

https://doi.org/10.1049/ip-gtd:20030982

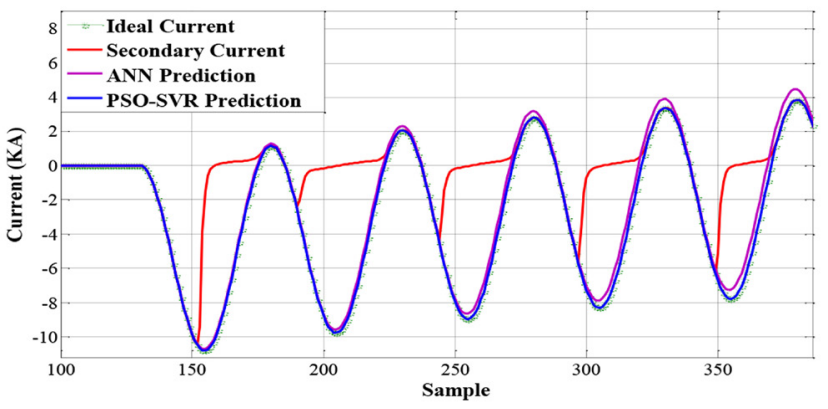

Fig. 8 Comparison of ANN and PSO-SVR method

[10] Bunyagul, T., Crossley, P., Gale, P. "Overcurrent protection using signals derived from saturated measurement CTs", In: Power Engineering Society Summer Meeting, Vancouver, BC, Canada, 2001, pp. 103-108.

https://doi.org/10.1109/PESS.2001.969993

[11] Bak, D. J., Dong, X. Z., Wang, B., Shi, S. X., Rebizant, W. "Approximation of Parameters of Fundamental Component Of Secondary Current Distorted by CT Saturation", In: 12th IET International Conference on Developments in Power System Protection, Copenhagen, Denmark, 2014.

https://doi.org/10.1049/cp.2014.0113

[12] Li, F., Li, Y., Aggarwal, R. K. "Combined wavelet transform and regression technique for secondary current compensation of current transformers", IEE Proceedings - Generation, Transmission and Distribution, 149(4), pp. 497-503, 2002.

https://doi.org/10.1049/ip-gtd:20020296

[13] Hong, Y.-Y., Chang-Chian, P.-C. "Detection and correction of distorted current transformer current using wavelet transform and artificial intelligence", IET Generation, Transmission and Distribution, 2(4), pp. 566-575, 2008.

https://doi.org/10.1049/iet-gtd:20070383

[14] Hong, Y.-Y., Wei, D.-W. "Compensation of Distorted Secondary Current Caused by Saturation and Remanence in a Current Transformer", IEEE Transactions on Power Delivery, 25(1), pp. 47-54, 2010.

https://doi.org/10.1109/TPWRD.2009.2034820

[15] Khorashadi-Zadeh, H., Sanaye-Pasand, M. "Correction of saturated current transformers secondary current using ANNs", IEEE Transactions on Power Delivery, 21(1), pp. 73-79, 2006. https://doi.org/10.1109/TPWRD.2005.858799

[16] Rebizant, W., Bejmert, D. "Current-Transformer Saturation Detection With Genetically Optimized Neural Networks", IEEE Transactions on Power Delivery, 22(2), pp. 820-827, 2007. https://doi.org/10.1109/TPWRD.2007.893363

[17] Ebadi, A., Hosseini, S. M., Abdoos, A. A. "لر كى ى

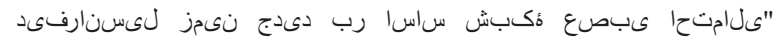
(Designing of a New Transformer Ground Differential Relay Based on Probabilistic Neural Network), Journal of Energy Engineering \& Management, 9(4), pp. 2-13, 2020. [online] Available at: http://energy.kashanu.ac.ir/article-1-1176-en.html [Accessed: 23 June 2020] (in Farsi) 
[18] Rebizant, W., Bejmert, D. "Current transformer saturation detection with genetically optimized neural networks", In: IEEE Russia Power Tech, St. Petersburg, Russia, 2005, pp. 1-6.

https://doi.org/10.1109/PTC.2005.4524417

[19] Vapnik, V. N. "The Nature of Statistical Learning Theory", Springer, New York, NY, USA, 2000.

https://doi.org/10.1007/978-1-4757-3264-1

[20] Kang, Y.-C., Lim, U.-J., Kang, S.-H., Crossley, P. "Compensation of the distortion in the secondary current caused by saturation and remanence in a CT", In: IEEE Power Engineering Society General Meeting, Denver, CO, USA, 2004. https://doi.org/10.1109/PES.2004.1372765

[21] Gil, M., Abdoos, A. A. "Intelligent busbar protection scheme based on combination of support vector machine and S-transform", IET Generation, Transmission \& Distribution, 11(8), pp. 2056-2064, 2017.

https://doi.org/10.1049/iet-gtd.2016.1686

[22] Ebadi, A., Hosseini, S. M., Abdoos, A. A. "A New Restricted Earth Fault Relay Based on Artificial Intelligence", IJE Transactions A: Basics, 32(1), pp. 62-70, 2019. https://doi.org/10.5829/ije.2019.32.01a.08

[23] Ebadi, A., Hosseini, S. M., Abdoos, A. A. "

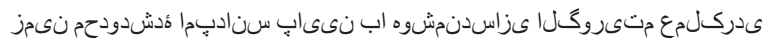

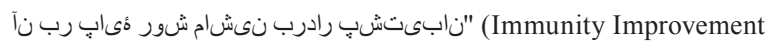
of a Low-Impedance Restricted Earth Fault Relay by Making its Mechanism Intelligent based on Support Vector Machine), Computational Intelligence in Electrical Engineering, pp. 29-40, 2018. (in Farsi) https://doi.org/10.22108/ISEE.2018.111464.1132

[24] Cortes, C., Vapnik, V. "Support-Vector Networks", Machine Learning, 20, pp. 273-297, 1995. https://doi.org/10.1023/A:1022627411411

[25] Yu, H., Kim, S. "SVM Tutorial - Classification, Regression and Ranking", In: Rozenberg, G., Bäck, T., Kok, J. N. (eds.) Handbook of Natural Computing, Springer, Berlin, Heidelberg, Germany, 2012, pp. 479-506.

https://doi.org/10.1007/978-3-540-92910-9_15

[26] Suganyadevi, M. V., Babulal, C. K. "Support Vector Regression Model for the prediction of Loadability Margin of a Power System", Applied Soft Computing, 24, pp. 304-315 2014. https://doi.org/10.1016/j.asoc.2014.07.015
[27] Wua, C.-H., Tzeng, G.-H., Lin, R.-H. "A Novel hybrid genetic algorithm for kernel function and parameter optimization in support vector regression", Expert Systems with Applications, 36(3), pp. 4725-4735, 2009.

https://doi.org/10.1016/j.eswa.2008.06.046

[28] Chen, X., Yang, J., Liang, J. "A flexible support vector machine for regression", Neural Computing and Applications, 21(8), pp. 2005-2013, 2012. https://doi.org/10.1007/s00521-011-0623-5

[29] Zhang, Y., Sun, H., Guo, Y. "Wind Power Prediction Based on PSO-SVR and Grey Combination Model", IEEE Access, 7, pp. 136254-136267, 2019. https://doi.org/10.1109/ACCESS.2019.2942012

[30] Zellagui, M., Benabid, R., Boudour, M., Chaghi, A. "Mixed Integer Optimization of IDMT Overcurrent Relays in the Presence of Wind Energy Farms Using PSO Algorithm", Periodica Polytechnica Electrical Engineering and Computer Science, 59(1), pp. 9-17, 2015. https://doi.org/10.3311/PPee.7525

[31] Mishra, S., Sagnika, S., Singh, S. S., Mishra, B. S. P. "Spectrum Allocation in Cognitive Radio: A PSO-based Approach", Periodica Polytechnica Electrical Engineering and Computer Science, 63(1), pp. 23-29, 2019. https://doi.org/10.3311/PPee.13074

[32] M'hamdi, B., Teguar, M., Tahar, B. "Optimal DG Unit Placement and Sizing in Radial Distribution Network for Power Loss Minimization and Voltage Stability Enhancement", Periodica Polytechnica Electrical Engineering and Computer Science, 64(2), pp. 157-169, 2020. https://doi.org/10.3311/PPee.15057

[33] Amer, M., Miloudi, A., Lakdja, F. "Optimal DTC Control Strategy of DFIG Using Variable Gain PI and Hysteresis Controllers Adjusted by PSO Algorithm", Periodica Polytechnica Electrical Engineering and Computer Science, 64(1), pp. 74-86, 2020. https://doi.org/10.3311/PPee.14237 